\title{
Development of Triamcinolone Acetonide Loaded Poly(lactic- co-glycolic acid) Dry Powder Inhaler Formulations for the Treatment of Asthma
}

\author{
Aysu Yurdasiper \\ Ege University, Faculty of Pharmacy, Department of Pharmaceutical Technology, Izmir, Türkiye. \\ Correspondence Author: Aysu Yurdasiper \\ E-mail: aysu.yurdasiper@ege.edu.tr \\ Received: $26.10 .2021 \quad$ Accepted: 01.12 .2021
}

\begin{abstract}
Objective: The objective of this study was to develop triamcinolone acetonide (TAA) loaded poly(lactide-co-glycolic acid) (PLGA) dry powder inhaler (DPI) formulations by using spray dryer and to characterize the formulations with reference to their appropriateness for pulmonary drug delivery.

Methods: PLGA dry powder inhalers containing TAA were produced in two stages. In the first step, PLGA microparticles were prepared by emulsion-solvent evaporation method and the DPI formulations were produced by the spray-drying process using mannitol and leucine. TAA loaded PLGA DPI was classified physicochemical properties. The in vitro dissolution test was carried out using modified USP apparatus 2. Aerosol performance was identified with next generation impactor.

Results: This study has demonstrated that TAA loaded PLGA DPI were effectively prepared. Scanning electron microscope illustrates the spherical shape, particle size (D50) was established to be between 2.7-3.1 $\mu \mathrm{m}$ and all formulations charged negatively. Special chemical interaction in the DPI was not observed by FT-IR. The in vitro aerosol performance study represented DPI formulations have the proper aerodynamic properties for targeting the lungs. The in vitro TAA release from DPI formulations decreased with increasing of PLGA concentration.

Conclusion: Developed PLGA dry powder inhaler formulations containing TAA have shown suitable aerodynamic characteristics to be administered to the lungs thereby could improve in the management of asthma by increasing TAA residency in the lungs for a prolonged period of time.
\end{abstract}

Keywords: Triamcinolone acetonide, pulmonary drug delivery, dry powder inhaler formulations, poly(lactide-co-glycolic acid), spray dryer

\section{INTRODUCTION}

Asthma is an illness caused by chronicinflammation of thelarge and small airways resulting in airway hyper-responsiveness and excessive mucous secretion. It is considered by various levels of symptoms of wheezing, shortness of breath and airflow obstruction (1). According to Global Initiative for Asthma (GINA) report 2021, GINA recommends that all adults and adolescents with asthma should obtain inhaled corticosteroids (ICS) containing controller treatment, to lessen the risk of severe exacerbations. However, there are side effects associated with this treatment, such as dysphonia and oral candidiasis. Up to $58 \%$ of patients report dysphonia at the same time as using inhaled corticosteroids (2).

The most crucial parameter defining the site of deposition of aerosol drugs within the respiratory tract is the particle characteristics of the aerosol. Good distribution all the way through the lung entails particles with an aerodynamic diameter between 1 and $5 \mu \mathrm{m}$ (3). The inhaled corticosteroid formulation contains a particle size larger than $5 \mu \mathrm{m}$, triggering local side effects. Subsequent to the inhalation, more or less, $10-60 \%$ of the inhaled dose is deposited in the lungs. The fraction of ICS (40-90\%) deposited in the oropharynx can result in local oropharyngeal side effects (4). Triamcinolone acetonide (TAA) is an effective corticosteroid used as first-line treatment of asthma in preventing asthma exacerbation. The local infections of the oropharynx with Candida albicans came about as a side effect in the commercial item for consumption containing TAA. The basis for this is that only $26 \%$ of the active substance reached the lungs in administration devoid of using spacer $(5,6)$.

Presently, DPI prepared with polymeric materials that endow with sustained release for local targeting is of great interest. Poly(lactic-co-glycolic acid) (PLGA) is a non-toxic, biodegradable and biocompatible polymer approved by Food and Drug Administration (FDA) and European Medicines 
Agency for drug delivery applications that have been expansively used in the encapsulation of drug molecules to augment their clinical efficacy (7).

The aim of the study was to develop and evaluate TAA loaded PLGA DPI for high pulmonary delivery, to increase the deposition and absorption of TAA in the deep lungs, thereby increasing the therapeutic efficacy. It is the first time demonstrated PLGA DPI formulation of TAA intended to pulmonary route. PLGA DPI containing TAA were characterized with respect to their morphology, particle size and size distribution, in vitro release and aerodynamic behaviour.

\section{METHODS}

\subsection{Materials}

TAA, leucine, polysorbate 80 , polysorbate 20 and sodium lauryl sulphate (SLS) were obtained from Sigma-Aldrich (Poole, U.K). PLGA (50:50) was purchased from Boehringer Ingelheim (Ingelheim Rhein, Germany). Mannitol was purchased from Roquette (Lestrem, France). Polyvinyl alcohol (PVA) (MW 20.000-30.000) was purchased from Acros (Geel, Belgium). Solvents were HPLC analytical grade (Merck, Darmstandt, Germany).

\subsection{Determination of TAA by HPLC}

An Agilent 1100 Series HPLC system (Santa Clara, CA, USA) equipped with thermostated column compartment and a UV absorbance detector were used for determination of TAA. The column temperature was controlled at $25^{\circ} \mathrm{C}$. The detection wavelength was set at $236 \mathrm{~nm}$. Chromatographic separation was carried out on the Thermo Scientific Hypersil ODS column $(250 \times 4.6 \mathrm{~mm}, 5 \mu \mathrm{m})$. The mobile phase was a mixture of water and acetonitrile [60:40] (8) and the retention time for TAA was $6.85 \mathrm{~min}$. The method was validated according to the guideline Q2 (R1) of ICH.

\subsection{Preparation of PLGA Microparticles Containing TAA}

TAA loaded PLGA microparticles were prepared by emulsification solvent evaporation technique. TAA and PLGA were dissolved in $2 \mathrm{~mL}$ acetone as internal phase and this organic solution was added to $20 \mathrm{~mL}$ external aqueous phase containing PVA $(1 \% \mathrm{w} / \mathrm{v})$ as a stabilizing agent. The resulted suspension was homogenized at $20.000 \mathrm{rpm}$ using a highspeed homogenizer (Ultra-Turrax, IKA T-18, Germany) for 2 minutes. Once the emulsion was formed, it was kept under constant agitation for 2 hours in order to remove the organic solvent by evaporation. The microparticles formed were separated by centrifugation at $30.000 \mathrm{rpm}$ (Optima MAX-XP Beckman Coulter, California, USA) for $1 \mathrm{~h}$ and the precipitated microparticles were washed twice by deionized water, using centrifuge ( $15.000 \mathrm{rpm}$ for 15 minutes) to eliminate remained
PVA. In conclusion, the obtained microparticles were dried at room temperature for 1 day and weighted. (9).

\subsection{Determination of Entrapment Efficiency}

TAA content in the PLGA microparticles was assessed by dispersing $3 \mathrm{mg}$ of microparticles in $5 \mathrm{ml}$ in $\mathrm{pH} 7.4$ PBS with $1 \%$ SLS. The dispersion was shaken for $24 \mathrm{hrs}$. Then, it was centrifuged at $15.000 \mathrm{rpm}$ for $30 \mathrm{~min}$ and the amount of TAA in the supernatant was analyzed by HPLC. The entrapment efficiency expressed in percentage were calculated according to Equation 1 ( $n=6)(10)$.

Drug entrapment efficiency $(\%)=$ (calculated drug concentration $/$ theoretical drug concentration) $\times 100$.......

\subsection{Preparation of PLGA Dry Powder Inhaler Formulations Containing TAA}

Microparticles (30\%), Mannitol (50\%) and leucine (20\%) were suspended in $50 \mathrm{~mL}$ deionized water then was spray-dried with constant stirring using a Büchi Nanospray dryer B-90 (Büchi Laboratory-Techniques, Flawil, Switzerland) under the following conditions: Nozzle: $5.5 \mu \mathrm{m}$ mesh, inlet temperature $80^{\circ} \mathrm{C}$, outlet temperature $40-42^{\circ} \mathrm{C}$, gas flow rate $116-120 \mathrm{~L} /$ min, Pressure: 31-36 mBar. The resultant spray dried powders were collected and yield percentage was calculated by the following Equation 2 (11).

Spray-drying yield $=$ weight of collected powder $/$ total mass of all ingredients of each formulations...

\subsection{Characterization of the Formulations}

\section{Differential scanning calorimetry Analysis}

Differential scanning calorimetry (DSC) of PLGA was performed using DSC-8000 (Perkin Elmer, Waltham, Massachusetts) equipped with a thermal analysis data acquisition software. The sample (between $3-6 \mathrm{mg}$ ) was heated in an aluminum pan using dry nitrogen as the effluent gas. The analysis was performed at a rate $10{ }^{\circ} \mathrm{C} / \mathrm{min}$ from $25^{\circ} \mathrm{C}$ to $300{ }^{\circ} \mathrm{C}$ temperature range under nitrogen flow of 20 $\mathrm{mL} / \mathrm{min}$.

\section{Thermogravimetric Analysis}

Thermogravimetric analysis (TGA) of the formulations was performed using a Perkin Elmer (TGA-4000, Waltham, Massachusetts) instrument. A ceramic pan was loaded with sample and heated from 25 to $250^{\circ} \mathrm{C}$ at a rate of $10^{\circ} \mathrm{C} / \mathrm{min}$ under dry nitrogen flowing at rate of $40 \mathrm{~mL} / \mathrm{min}$.

\section{Fourier-Transform Infrared Spectroscopy}

Fourier-transform infrared (FT-IR) spectra of the samples were obtained using a Perkin Elmer Spectrum 100 FT-IR spectrometer attached with an attenuated total reflectance accessory (Perkin Elmer Spectrum 100, Waltham, 
Massachusetts) with wave numbers ranging from $4000 \mathrm{~cm}^{-1}$ to $650 \mathrm{~cm}^{-1}$, using four scans with a resolution of $4 \mathrm{~cm}^{-1}$.

\section{Particle Morphology}

The surface morphology of the formulations was evaluated using scanning electron microscopy (SEM, Quanta 250 FEG, Hillsboro, OR) at $15 \mathrm{kV}$, with magnifications of $10.000 \times$ and 25.000x.

\section{Particle Size Distribution and Surface Charge}

The size of the particles was determined by laser diffraction with a dry sample dispersion system (Mastersizer 3000, Aero $\mathrm{S}$, Malvern instruments, UK). D50 represent the equivalent diameters at $50 \%$ cumulative volume of the particles. The size distribution Span was calculated according to Equation (3). Zeta potential (ZP) of formulations were measured by photon correlation spectroscopy (Nano ZS, Malvern Instruments, UK) $(n=3)$.

Span = D90-D10/D50.

\subsection{Aerodynamic Particle Size Analysis}

The aerodynamic particle size whose allocation has been identified was analyzed by using a Next Generation Impactor (NGI, Copley Scientific Ltd., Nottingham, UK) and a standard pharmacopeial procedure was used (USP 39 (601). A dry powder having an inhalation apparatus (Aerolizer ${ }^{\circledR}$, Novartis, Switzerland) was filled with a No. 3 hydroxypropyl methylcellulose capsule (Capsugel, France). The flow rate was adapted to a pressure drop of $4 \mathrm{kPa}$, resulting in a flow rate of $100 \mathrm{~L} / \mathrm{min}$ for $2.3 \mathrm{~s}$. Following aerosolization progression, the disseminated powders remained in the capsule and the powder deposited in the entire the parts of NGI were gathered using mobile phase and analyzed by HPLC. The fine particle fraction (FPF), emitted dose (ED), respirable fraction (RF), mass median aerodynamic diameter (MMAD) and geometric standard deviation (GSD) were determined. The emitted dose (ED), fine particle fraction (FPF), and respirable fraction (RF) were calculated as follows (Equation $(4,5,6)$ :

Emitted dose $($ ED\%) $=($ Initial mass in capsules - Final mass remaining in capsules)/Initial mass in capsules $\times 100 \%$.

(4)

Fine particle fraction (FPF\%) $=$ Mass deposited on stages 2 through 7/ Initial particle mass loaded into capsules $\times$ $100 \%$

Respirable fraction (RF \%) = Mass deposited on stages 2 through $7 /$ Initial particle mass on all stages $\times 100 \%$. amount of TAA were added to $5 \mathrm{~mL}$ solutions and shaken for $24 \mathrm{hrs}$ at room temperature. Samples centrifuged at 10.000 rpm for $10 \mathrm{~min}(n=6)$. The amount of TAA in the samples was analyzed by HPLC.

\subsection{In Vitro Dissolution Studies}

Dissolution testing was performed on $15 \mathrm{mg}$ spray dried powders using "paddle over disc" method previously described (12). For the dissolution test, NGI was run to ensure that sufficient formulation dose was accumulated at stage 2-3. After dose collection was completed, part of the dissolution cup was removed and a $55 \mathrm{~mm}$ diameter presoaked polycarbonate membrane was placed over it. 300 $\mathrm{ml}$ phosphate buffer $\mathrm{pH} 7.4$ containing $1 \%(\mathrm{w} / \mathrm{v})$ of SLS was used as dissolution media. Dissolution testing was performed in accordance with sink conditions, at $37 \pm 1{ }^{\circ} \mathrm{C}$ with a paddle rotating speed of $75 \pm 2 \mathrm{rpm}$. Six samples from each formulation (F1, F2 and F3) were studied. $1 \mathrm{ml}$ of sample was withdrawn at each time interval and replaced with fresh dissolution medium to maintain the sink condition. Samples $(1 \mathrm{~mL})$ were analyzed by HPLC.

\subsection{Statistical Analysis}

For drug release data one-way analysis of variance (ANOVA) and post hoc Tukey-Kramer multiple comparisons were performed (GraphPad Prism 5, La Jolla, USA). Data were shown as mean \pm standard error of mean (S.E.M.) and $p<0.05$ was accepted as statistically significant.

\section{RESULTS}

\subsection{Preparation and Physicochemical Properties of PLGA Dry Powder Inhaler Formulations Containing TAA}

TAA loaded PLGA microparticles were prepared effectively by emulsion-solvent evaporation method and the DPI formulations were formed subsequent to spray-drying process using mannitol and leucine as DPI carriers. Unclustered and comparatively monodisperse TAA loaded PLGA DPI were attained. SEM images of DPI formulations displayed almost spherical structures with smooth surfaces devoid of pores or voids (Fig. 1). As given in Table 1, the entrapment efficiency of PLGA microparticles increased in conjunction with augmenting polymer concentration. The entrapment efficiency for all microparticle formulations was greater than $41 \%$ and the uppermost entrapment efficiency was noted down in the F1 formulation with $54.5 \%$.
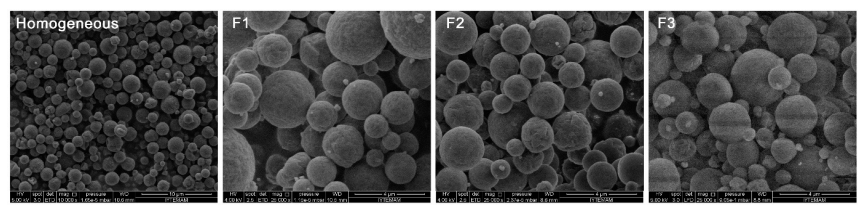

Figure 1. SEM micrographs of DPI formulations. 
It has been confirmed that gathering microparticles by spray drying is proficient and convenient, and furthermore, it increases the percent yield (13). The process yields for DPI formulations were between $48.7 \%$ and $64.6 \%$, processing of fewer polymers given higher percent yield.

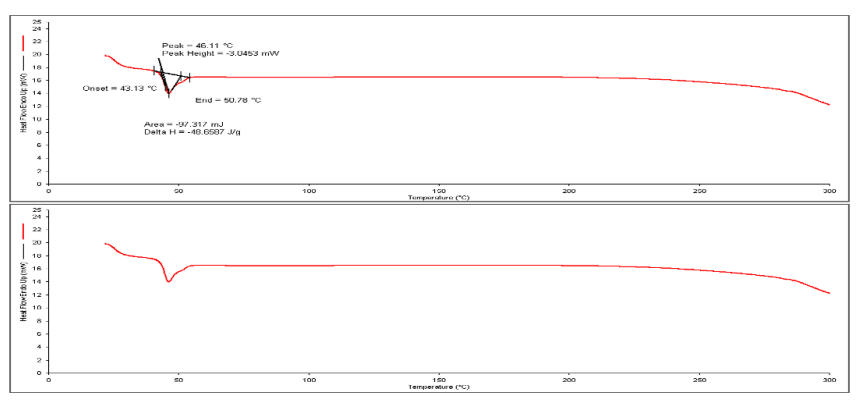

Figure 2. DSC thermogram of PLGA

Figure 2 shows the DSC thermogram of PLGA and the amorphous nature of PLGA indicated an endothermic peak at $46.11{ }^{\circ} \mathrm{C}$ corresponding to the polymer transition temperature (Tg). The water content of DPI formulations was $2 \%-2.3 \%$ indicating that water was removed during the spray-drying process.
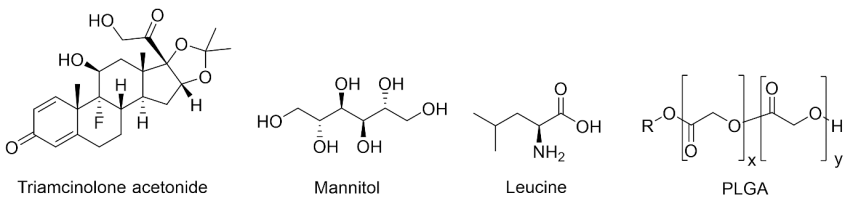

Figure 3. Chemical structures of the compounds used in the DPI formulations

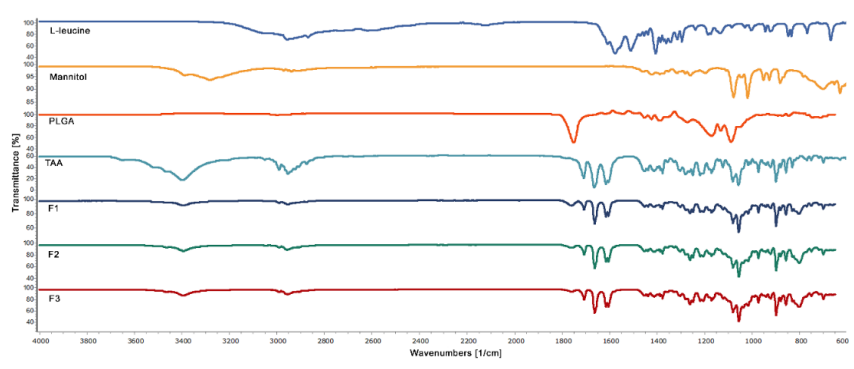

Figure 4. FT-IR spectra of the formulations and formulation components
The chemical structures of the compounds (Fig. 3) were assessed by using FT-IR analysis (Fig. 4). The FT-IR spectra of PLGA displayed a carbonyl stretching band at $1750 \mathrm{~cm}^{-1}$, $\mathrm{C}(=\mathrm{O})-\mathrm{O}$ stretching band at $1172 \mathrm{~cm}^{-1}$ and $\mathrm{O}-\mathrm{C}-\mathrm{C}$ stretching band at $1086 \mathrm{~cm}^{-1}$ thus indicating the presence of ester functional group. Other bands observed at 2999-2952.2, 1423 and $1386 \mathrm{~cm}^{-1}$ can be assigned as $\mathrm{C}-\mathrm{H}$ stretching, $\mathrm{C}-\mathrm{H}$ asymmetric and symmetric bending bands respectively. The FT-IR spectra of mannitol exhibited a hydroxyl group stretching band and an out of plane $-\mathrm{OH}$ bending band at $3283 \mathrm{~cm}^{-1}$ and $700.99 \mathrm{~cm}^{-1}$, respectively. The C-O stretching bands for mannitol were also observed as two sharp bands at around 1080 and $1020 \mathrm{~cm}^{-1}$ region. Additionally, two bands observed at around 1419 and $1350 \mathrm{~cm}^{-1}$ region are a result of the coupling vibrations between $\mathrm{OH}$ in-plane bending and $\mathrm{C}-\mathrm{H}$ wagging vibrations which can be confirmative for primary and secondary alcohol groups in mannitol structure. The observed $-\mathrm{NH}^{+}$stretching bands $\left(3062 \mathrm{~cm}^{-1}\right)$, asymmetric $\left(1605 \mathrm{~cm}^{-1}\right)$ and symmetric $-\mathrm{NH}^{+}$bending vibrations (1512 $\left.\mathrm{cm}^{-1}\right)$, asymmetric $\left(1575 \mathrm{~cm}^{-1}\right)$ and symmetric $\left(1405 \mathrm{~cm}^{-}\right.$ $\left.{ }^{1}\right) \mathrm{C}=\mathrm{O}$ stretching bands in FT-IR spectra of leucine were in agreement with the literature $\mathrm{C}=\mathrm{O}$ stretching bands in $\mathrm{FT}$-IR spectra of leucine were in agreement with the literature (14). The latter also displayed C-N stretching, $\mathrm{O}-\mathrm{H}$ out of plane bending and $\mathrm{N}-\mathrm{H}$ rocking bands at 1296, 920 and $666 \mathrm{~cm}$ 1 , respectively. TAA, has $-\mathrm{OH}$ stretching band at $3395 \mathrm{~cm}^{-1}$ and $\mathrm{C}-\mathrm{H}$ stretching bands at $2991.7-2873.5 \mathrm{~cm}^{-1}$ as expected. The dioxole residue of the molecule yielded with a $\mathrm{C}-\mathrm{O}-\mathrm{C}$ asymmetric stretching band at $1055 \mathrm{~cm}^{-1}$ whereas tertiary and secondary alcohol groups produce $\mathrm{C}-\mathrm{O}$ stretching and $\mathrm{O}-\mathrm{H}$ out of plane bending vibrations at 1218, 1079 and 700 $\mathrm{cm}^{-1}$, respectively.

The particle size distribution and ZP values of the DPI formulations were presented in Table 1. All formulations exhibited relatively narrow range distributions of $<5 \mu \mathrm{m}$ and with spans in the range of $2.19 \pm 0.13-2.73 \pm 0.39$, which were appropriate for DPI formulations. Zeta potential measurements make available information about particle stability in the formulation. The higher value indicates better stability due to repulsion between particles and homogeneity of the system on account of reducing aggregation. ZP values were negative and varied between $-19.4 \pm 0.39$ and $-25.4 \pm 2.7$. The result showed that increasing of TAA in the formulation increased the negative zeta potential. Similar results were reported by Yildiz et al. (15).

Table 1. Physical characterization of formulations.

\begin{tabular}{|c|c|c|c|c|c|c|c|}
\hline Code & TAA:PLGA & $\begin{array}{c}\text { D50 } \pm S D \\
(\mu \mathrm{m})\end{array}$ & Span & $\begin{array}{c}\mathrm{ZP} \pm S D \\
(\mathrm{mV})\end{array}$ & $\begin{array}{c}\text { Process yield } \pm \text { SD } \\
\text { (\%) }\end{array}$ & $\begin{array}{c}\text { Entrapment } \\
\text { efficiency } \pm \text { SD } \\
(\%)\end{array}$ & $\begin{array}{c}\text { Water } \\
\text { content } \pm \text { SD } \\
(\%)\end{array}$ \\
\hline F1 & 10:1 & $3.1 \pm 0.3$ & $2.73 \pm 0.3$ & $-19.4 \pm 2.6$ & $48.7 \pm 2.9$ & $54.5 \pm 2.2$ & $2.3 \pm 0.4$ \\
\hline F2 & $20: 1$ & $2.8 \pm 0.4$ & $2.34 \pm 0.2$ & $-22.6 \pm 1.8$ & $59.4 \pm 3.2$ & $47.9 \pm 2.8$ & $2 \pm 0.6$ \\
\hline F3 & $30: 1$ & $2.7 \pm 0.1$ & $2.19 \pm 0.1$ & $-25.4 \pm 2.7$ & $64.6 \pm 2.1$ & $42.3 \pm 1.4$ & $2.1 \pm 0.2$ \\
\hline
\end{tabular}

TAA: triamcinolone acetonide; PLGA: Poly (lactic-co-glycolic acid); ZP: zeta potential, SD: standard deviation. 


\subsection{Aerodynamic Particle Size Analysis}

The aerodynamic characteristics of the formulations analyzed with NGI are shown in Table 2. MMAD assessment of between 1 and $5 \mu \mathrm{m}$ is preferable for targeting the respiratory region of the human lung (16). MMAD and FPF varied from 2.46 \pm 0.4 to $4.1 \pm 0.3 \mu \mathrm{m}$ and $39.42 \pm 1.4$ to $54.17 \pm 1.3 \%$, respectively. Despite the fact that all formulations were executed beneath the similar spray-drying circumstances, F1 showed the smallest MMAD. Likewise, FPF of FI was considerably superior to that of F2 and F3. Even though the quantity of polymer is declined by half, RF values of F1 and F2 was near to each other. ED uniformity for all formulations was in the high range $(93.68 \pm 1.28-98.31 \pm 1.77 \%)$ for pulmonary route. The GSD (1.73 to 1.91) values of formulations pointed out that the formulations are suitable for pulmonary delivery (17).

Table 2. The aerodynamic characteristics of DPI formulations measured by NGI.

\begin{tabular}{|c|c|c|c|c|c|}
\hline Formulations & $\begin{array}{c}\text { FPF } \pm S D \\
(\%)\end{array}$ & $\begin{array}{c}E D \pm S D \\
(\%)\end{array}$ & $\begin{array}{c}\text { RF } \pm S D \\
(\%)\end{array}$ & $\begin{array}{c}\text { MMAD } \pm S D \\
(\mu \mathrm{m})\end{array}$ & GSD $\pm S D$ \\
\hline F1 & $54.17 \pm 1.3$ & $98.31 \pm 3.9$ & $78.51 \pm 2.7$ & $2.46 \pm 0.4$ & $1.73 \pm 0.2$ \\
\hline F2 & $43.39 \pm 2.3$ & $97.24 \pm 2.4$ & $78.06 \pm 3.4$ & $3.77 \pm 0.8$ & $1.91 \pm 0.5$ \\
\hline F3 & $39.42 \pm 1.4$ & $93.68 \pm 2.8$ & $70.92 \pm 2.9$ & $4.1 \pm 0.3$ & $1.83 \pm 0.4$ \\
\hline
\end{tabular}

FPF: fine particle fraction; ED: emitted dose; MMAD: mass median aerodynamic diameter; GSD: geometric standard deviation, SD: standard deviation.

\subsection{Solubility Studies}

Solubility studies aimed to determine the appropriate dissolution media that provides sufficient sink conditions for the release of TAA from DPI formulations. PB pH $7.4+1 \%$ $(w / v)$ SLS exhibited maximum solubility for TAA. Results are depicted in Table 3.

Table 3. Solubility of TAA in different dissolution media.

\begin{tabular}{|lc|}
\multicolumn{1}{|c|}{ Dissolution media } & $\begin{array}{c}\text { Solubility } \\
(\mu \mathrm{m} / \mathrm{mL}) \pm \text { SD }\end{array}$ \\
\hline Deionized water & $17.28 \pm 0.7$ \\
\hline Phosphate buffer $\mathrm{pH} 7.4$ & $18.76 \pm 1.1$ \\
\hline $\begin{array}{l}\text { Phosphate buffer } \mathrm{pH} 7.4+1 \%(\mathrm{w} / \mathrm{v}) \text { sodium lauryl } \\
\text { sulphate }\end{array}$ & $833.41 \pm 12.9$ \\
\hline $\begin{array}{l}\text { Phosphate buffer } \mathrm{pH} 7.4+2 \%(\mathrm{w} / \mathrm{v}) \text { polysorbate } 80 \\
\text { Phosphate buffer } \mathrm{pH} 7.4+2 \%(\mathrm{w} / \mathrm{v}) \text { polysorbate } 20\end{array}$ & $162.23 \pm 7.4$ \\
\hline
\end{tabular}

$S D$ : standard deviation.

\subsection{In Vitro Dissolution Studies}

Figure 5 illustrates in vitro release profiles of TAA from DPI formulations. All formulations showed a biphasic release profile with an initial burst release followed by slower release (18). The release percentages of TAA were $37.6 \%$ for F1, $46.01 \%$ for $F 2$, and $62.93 \%$ for $F 3$ at 12 hrs.

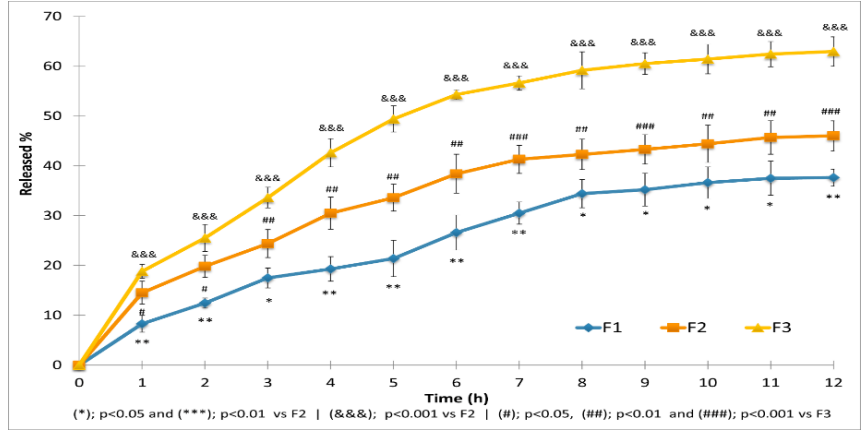

Figure 5. In vitro release profiles of TAA in phosphate buffer, $p H 7.4$ $+1 \% S L S$.

\section{DISCUSSION}

Images were acquired using an SEM, as provided in Fig. 1, DPI formulations were fairly spherical, smooth, and regular. The drug: polymer concentrations of which the formulations did not radically affect the morphologic properties. In addition, the outlet temperature of spray dryer was below the glass transition temperature of PLGA $\left(\operatorname{Tg} \approx 47^{\circ} \mathrm{C}\right)$ hereby, the structural forms of the microparticles were preserved and prevented from accumulating on the wall by softening of microparticles (19). PLGA concentration in the oil phase is a key factor having an effect on the entrapment efficiency of PLGA microparticles containing TAA. Microparticles were prepared using PLGA by altering concentration as given in Table 1. Increase in concentration of polymer led to an improvement in drug entrapment efficiency (from 42.3\% to $54.5 \%$ ) (20). Panyam et al. (21) indicated that as PLGA amount increases in the formulation, hydrophobic drugs will mix with PLGA more and thus higher drug entrapment efficiency will be achieved. The percentage of water content results was about to each other in all formulations. Generally, low moisture is expected in the spray-dried powders as a high amount of moisture can decrease particle dispersion by the formation of interparticle forces. One of the reasons for the low moisture content in formulations is that mannitol has a lower moisture absorption capacity than lactose and glucose, improving the flow properties of powders for inhalation (22).

Considering the IR spectra of all formulations, the overlapping of carbonyl enlarging band of PLGA, leucine and TAA yielded with a broad band at around $1700 \mathrm{~cm}^{-1}$. Conversely, $\mathrm{N}-\mathrm{H}$ rocking and $\mathrm{C}-\mathrm{O}-\mathrm{H}$ in plane bending vibrations were probative for leucine, while vinyllic stretching bands and out of plane bending of $-\mathrm{OH}$ were confirmative for TAA and mannitol, respectively. In brief, the FT-IR spectra of the three PLGA matrix (F1, F2, F3) unveiled the predictable vibrational bands and didn't demonstrate any supplementary or unexpected absorbsions thus suggesting physical entrapment of TAA within the PLGA matrix. The FT-IR analysis was employed as to accumulate information about the probable interactions 
of the compounds utilized for the formulations of the study. Since TAA loaded PLGA DPI didn't exhibited any additional vibration bands in the FT-IR spectra, that there wasn't any molecular interaction between the compounds used for the formulation can be concluded.

It is well known that particles less than $5 \mu \mathrm{m}$ can be dispersed deep into the smaller airways and the penetration concerned correlates well with a good quality clinical necessity to a treatment which is found locally. All DPI formulations possessed small D50 values with narrow span in particle size distribution which was suitable for inhalation. The SEM images were confirmed by the results obtained. Moreover, increasing the concentration of PLGA will enlarge the particle size of microspheres. The increase in the amount of PLGA increases the viscosity of the solutions, resulting in the formation of larger particles. Similar results established by Han et al. (23). The charge on the respiratory system is negative owing to mucin (24). Negative charge on the respiratory system and negative charged DPI microparticles could repulse each other and microparticles can advance to the deep lung region without collapsing in the upper airway. In a similar study, Mali et al. (25) studied on budesonide DPI formulation which has shown negative surface charge of $-17.5 \mathrm{mV}$.

It is essential to assure that the microparticles might be potentially delivered to the pulmonary route as the target organ. Thus, FPF is an eminent parameter for identifying the aerodynamic behavior of spray dried particles (26). In the present study, an augmented lung deposition of F1was observed as measured up to F2 and F3. This may be correlated to the presence of higher polymer ratio, which reduced the agglomeration of the particles. F1 was easily dispersed to aerosols characterized by high FPF. This situation is additionally confirmed by the data taken from FPF of F3. As FPF of F3 value was the lowest among formulations, indicating strong cohesiveness between the particles by reason of having the lowest amount of polymer. Furthermore, presence of L-leucine in formulations brings about decline in particles interaction and augment in fine particle fraction. The motive why l-leucine is able to efficiently increase the aerosolization performance of the spray dried powders might be attributed to its anti-adherent and surface active properties, consequently affecting the aerosolization properties of the powders (27). Besides the fine particle fraction for lung deposition of a drug formulation, another important factor is mass distribution of the MMAD. Likewise, MMAD of F3 value was higher than F1 and F2. Devrim et al. (28) suggested that the increase in the MMAD values was related to the aggregate formation by the particles during the aerosolization because of the effect of cohesive forces. GSD values above 1.5 all powders can be regarded as polydisperse, which is typical for most pharmaceutical aerosols (29). The GSD values were in the range of 1.73 to 1.91 , all formulations showed low GSD values, which indicated that the aerosolized particles were narrowly distributed in size. Based on the experimental data, the zeta potential of formulations has less impact on FPF. F1 formulation has the highest FPF and lowest MMAD and GSD values among all formulations. The present results indicate that $\mathrm{F} 1$ could reach the lungs in high amounts.

According to solubility studies, TAA was most dissolved in phosphate buffer pH7.4+ $1 \%$ SLS, therefore, phosphate buffer $\mathrm{pH} 7.4+1 \%$ SLS was used as dissolution media to ensure the sink condition. FDA has allowed some surfactants, such as SLS and polysorbates, to be used as dissolution enhancers. However, FDA has approved the use of SLS up to $2 \%$ in dissolution media (30). Considering in vitro dissolution study, two stages could be determined according to the rate of drug release; phase $I$ is defined as burst release and phase II shows sustained release (31). Owing to TAA released from the pores near the particle surface, burst release could have occurred within 2 hours in all formulations. F1, F2 and F3 showed an initial drug release of $12.46 \pm 1.01 \%$, $19.82 \pm 2.2 \%$ and $25.5 \pm 2.7 \%$ respectively. Thereafter, the drug release rate depends on the molecular diffusion rate in the polymer matrix or erosion from the particle matrix and this is attributed to phase II. During this stage, TAA encapsulated inside the PLGA microparticles could be released out slowly. The rate of release of the drug from each DPI formulation differed owing to the amount of PLGA in formulations. The rate limiting step is the amount of PLGA in the formulations. As expected, the release rate slowed down as the PLGA ratio in the formulations increased (32). Hence, F3 with the lowest PLGA ratio reached the highest drug release $(62.93 \pm 2.86 \%)$ among formulations. In other words, TAA was hardly released from $\mathrm{F} 1$ because the degradation rate of PLGA in F1 was slower than F2 and F3. Also, one of the reasons leading to slow diffusion of TAA from polymeric matrix might be low swelling of the polymer in the dissolution medium. Among the formulations, F1 has the highest polymer concentration and increasing the polymer concentration in the formulation causes a decrease in the swelling rate. Since the amount of polymer in the $\mathrm{F} 3$ formulation is the least compared to the F1 and F2 formulations, it is thought that the F3 formulation has the highest swelling capacity in the dissolution medium, so the release pattern is quite different from the F1 and F2 formulations. As observed, there was a significant rise in the release rate of $\mathrm{F} 3$ among formulations.

In terms of statistical analysis, at all time points, the drug release was different from each other, which was statistically higher in the F3 formulation than in the F2 and F1 formulations, and in the F2 formulation than in the F1 formulation.

\section{CONCLUSION}

TAA-loaded PLGA DPI prepared and evaluated in this study could provide prolonged drug delivery to the lungs, by which it will not only increase the therapeutic efficacy as decreasing the therapeutic dose but also endow with a useful inhaler dosage form for patients suffering from asthma. 


\section{Conflict of Interest}

The author stated that there are no conflicts of interest regarding the publication of this article.

\section{Acknowledgments}

The author would like to acknowledge Assist. Prof. Dr. Özlem Akgül for the interpretation of FT-IR experiments and Dr. Mehmet Ali Ege, Assoc. Prof. Dr. Aylin Arıcı for assisting in performing the statistical analyses.

\section{Funding}

This work was supported by financial support from Ege University Project Coordination Center (Projects 13/ECZ/017) and the author acknowledges Pharmaceutical Sciences Research Centre (FABAL) of Ege University, Faculty of Pharmacy for enabling to use of its laboratory instruments.

\section{REFERENCES}

[1] Quirce S, Heffler E, Nenasheva N, Demoly P, Menzies-Gow A, Moreira-Jorge A, Nissen F, Hanania NA. Revisiting late-onset asthma: Clinical characteristics and association with allergy. J Asthma Allergy 2020;13:743-752.

[2] Ye Q, He XO, D'Urzo A. A Review on the safety and efficacy of inhaled corticosteroids in the management of asthma. Pulm Ther 2017;3:1-18.

[3] Zanen P, Go LT, Lammers JWJ. The optimal particle-size for betaadrenergic aerosols in mild asthmatics. Int J Pharmaceutics 1994;107:211-217.

[4] Galvan CA, Guarderas JC. Practical considerations for dysphonia caused by inhaled corticosteroids. Mayo Clin Proc 2012;87(9):901-904.

[5] Hirst PH, Pitcairn GR, Richards JC, Rohatagi S, Gillen MS, Newman SP. Deposition and pharmacokinetics of an HFA formulation of triamcinolone acetonide delivered by pressurized metered dose inhaler. J Aerosol Med 2001;14(2), 155-165.

[6] Argenti D, Shah B, Heald D. A pharmacokinetic study to evaluate the absolute bioavailability of triamcinolone acetonide following inhalation administration. J Clin Pharmacology 1999;39:695-702.

[7] Eroglu H, Haidar MK, Nemutlu E, Ozturk S, Bayram C, Ulubayram K, Oner L. Dual release behavior of atorvastatin and alpha-lipoic acid from PLGA microspheres for the combination therapy in peripheral nerve injury. J Drug Del Sci Tech 2017;39:455-466.

[8] Fantini A, Padula C, Nicoli S, Pescina S, Santi P. The role of vehicle metamorphosis on triamcinolone acetonide delivery to the skin from microemulsions. Int J of Pharm 2019;565:3340.

[9] Hamishehkar H, Emami J, Najafabadi AR, Gilani K, Minaiyan M, Hassanzadeh K, Mahdavi H, Koohsoltani M, Nokhodchi A. Pharmacokinetics and pharmacodynamics of controlled release insulin loaded PLGA microcapsules using dry powder inhaler in diabetic rats. Biopharm Drug Dispos 2010;31:189201.
[10] Yalcin TE, Tamer SI, Takka S. Effect of organic solvents on gemcitabine loaded PLGA nanoparticles. FABAD J Pharm Sci 2017;42:15-19.

[11] Scherließ R, Janke J. Preparation of poly-lactic-coglycolic acid nanoparticles in a dry powder formulation for pulmonary antigen delivery. Pharmaceutics 2021;13:1196.

[12] Son YJ, McConville J.T. Development of a standardized dissolution test method for inhaled pharmaceutical formulations. Int J Pharm 2009;382(1-2):15-22.

[13] Dantas D, Pasquali MA, Cavalcanti-Mata M, Duarte ME, Lisboa $\mathrm{HM}$. Influence of spray drying conditions on the properties of avocado powder drink. Food Chem 2018;266:284-291.

[14] Silverstein RM, Webster FX, Kiemle DJ. Spectrometric identification of organic compounds. John Wiley \& Sons, Inc., editors. Hoboken 2005.p.1-550.

[15] Yildiz A, John E, Ozsoy Y, Araman A, Birchall JC, Broadley KJ, Gumbleton M. Inhaled extended-release microparticles of heparin elicit improved pulmonary pharmacodynamics against antigen-mediated airway hyper-reactivity and inflammation. J Control Release 2012;162(2):456-463.

[16] Lu W, Rades T, Rantanen J, Yang M. Inhalable co-amorphous budesonide-arginine dry powders prepared by spray drying. Int J Pharmaceutics 2019;565:1-8.

[17] Momin MAM, Tucker IG, Doyle CS, Denman JA, Das SC. Manipulation of spray-drying conditions to develop dry powder particles with surfaces enriched in hydrophobic material to achieve high aerosolization of a hygroscopic drug. Int J Pharmaceutics 2018;543(1-2):318-327.

[18] Varshosaz J, Taymouri S, Hamishehkar H, Vatankhah R, Yaghubi $\mathrm{S}$. Development of dry powder inhaler containing tadalafilloaded PLGA nanoparticles, Res Pharm Sci 2017;12(3):222232.

[19] D'Aurizio E, Van Nostrum CF, Van Steenbergen MJ, Sozio P, Siepmann F, Siepmann J, Hennink WE, Di Stefano A. Preparation and characterization of poly(lactic-co-glycolic acid) microspheres loaded with a labile antiparkinson prodrug. Int J Pharm 2011;409:289-296.

[20] Su Y, Zhang B, Sun R, Liu W, Zhu Q, Zhang X, Wang R, Chen C. PLGA-based biodegradable microspheres in drug delivery: recent advances in research and application. Drug Delivery 2021;28:(1)1397-1418.

[21] Panyam J, William D, Dash A, Leslie-Pelecky D, Labhasetwar V. Solid-state solubility influences encapsulation and release of hydrophobic drugs from PLGA/PLA nanoparticles. J of Pharm Sci 2004;93(7):1804-1814.

[22] Price R, Young PM, Edge S, Staniforth JN. The influence of relative humidity on particulate interactions in carrier-based dry powder inhaler formulations. Int. J Pharm 2002;246:4759.

[23] Han FY, Thurecht KJ, Whittaker AK, Smith MT. Bioerodable PLGA-based microparticles for producing sustained-release drug formulations and strategies for improving drug loading. Front Pharmacol 2016;7(185):1-11.

[24] Kim J, Lee B, Lee J, Ji M, Park CS, Lee J, Kang M, Kim J, Jin M, Kim $\mathrm{HH}$. N-Glycan modifications with negative charge in a natural polymer mucin from bovine submaxillary glands, and their structural role. Polymers (Basel) 2020;13(1):103.

[25] Mali AJ, Pawar AP, Purohit RN. Development of budesonide loaded biopolymer based dry powder inhaler: optimization, in vitro deposition, and cytotoxicity study. Journal of Pharmaceutics 2014; Article ID 795371. 
[26] Cayli YA, Sahin S, Buttini F, Balducci AG, Montanari S, Vural I, Oner L. Dry powders for the inhalation of ciprofloxacin or levofloxacin combined with a mucolytic agent for cystic fibrosis patients. Drug Dev Ind Pharm 2017;43(8):1378-1389.

[27] Alhajj N, O'Reilly NJ, Cathcart H. Leucine as an excipient in spray dried powder for inhalation. Drug Discovery Today 2021;26(10):2384-2396.

[28] Devrim B, Bozkir A, Canefe K. Preparation and evaluation of poly(lactic-co-glycolic acid) microparticles as a carrier for pulmonary delivery of recombinant human interleukin-2: II. In vitro studies on aerodynamic properties of dry powder inhaler formulations, Drug Dev Ind Pharm 2011;37(11):1376-1386.

[29] Scichilone N, Spatafora M, Battaglia S, Arrigo R, Benfante A, Bellia V. Lung penetration and patient adherence considerations in the management of asthma: role of extrafine formulations. J Asthma Allergy 2013;6:11-21.

[30] FDA, Dissolution methods. https://www.accessdata.fda. gov/scripts/cder/dissolution/dsp_getalldata.cfm (accessed November 23, 2021).

[31] Zhang Y, Zhang R, Illangakoon UE, Harker AH, Thrasivoulou C, Parhizkar M, Edirisinghe M, Luo CJ. Copolymer composition and nanoparticle configuration enhance in vitro drug release behavior of poorly water-soluble progesterone for oral formulations. Int J Nanomedicine 2020;15:5389-5403.

[32] Yao S, Liu H, Yu S, Li Y, Wang X, Wang L, Drug-nanoencapsulated PLGA microspheres prepared by emulsion electrospray with controlled release behavior. Regenerative Biomaterials 2016;3(5):309-317.

How to cite this article: Yurdasiper A. Development of Triamcinolone Acetonide Loaded Poly(lactic-co-glycolic acid) Dry Powder Inhaler Formulations for the Treatment of Asthma. Clin Exp Health Sci 2022; 12: 249-256. DOI: 10.33808/clinexphealthsci.1015166 